\title{
Efficient approximate scaling of spherical functions in the Fourier domain with generalization to hyperspheres
}

\author{
Ivan Dokmanić, Student Member, IEEE, Davor Petrinović, Member, IEEE
}

\begin{abstract}
We propose a simple model for approximate scaling of spherical functions in the Fourier domain. The proposed scaling model is analogous to the scaling property of the classical Euclidean Fourier transform. Spherical scaling is used for example in spherical wavelet transform and filter banks or illumination in computer graphics. Since the function that requires scaling is often represented in the Fourier domain, our method is of significant interest. Furthermore, we extend the result to higher-dimensional spheres. We show how this model follows naturally from consideration of a hypothetical continuous spectrum. Experiments confirm the applicability of the proposed method for several signal classes. The proposed algorithm is compared to an existing linear operator formulation.
\end{abstract}

Index Terms-sphere, scaling, spherical harmonics, $n$-sphere, hyperspherical harmonics

\section{INTRODUCTION}

In many cases it is convenient to describe a spherical function using its Fourier coefficients, often because spherical harmonics of a given order span a rotationally invariant subspace in the space of square integrable functions on the unit sphere, $L^{2}\left(S^{2}\right)$. Various applications require scaling (dilating or contracting) of the function on a sphere and if the function is represented by its Fourier coefficients, it would be beneficial to perform the scaling directly in the Fourier domain. See for example spherical wavelets, [1], [2], spherical filter banks, [3], illumination in computer graphics [4] or spherical point density estimation, [5], [6]. Spectral computation is further facilitated by the development of fast spherical transform algorithms [7], [8] analogous to the Euclidean fast Fourier transform.

A linear operator formulation of spectral domain scaling has been proposed in [4]. We propose an approximate method that is very simple in comparison to the existing methods - it involves resampling of the spectrum, and the computational cost is equal to the cost of the resampling step. Our method is based on a neat property that we came across during our previous work on $n$-spherical convolution and density estimation.

It is known that the functions on compact spaces such as the sphere have a discrete Fourier transform. We propose an approximate continuous spectrum model that mimics the scaling property of a classical Fourier transform in the context of Euclidean signal processing. An analogy may be drawn with the function on a circle: it is natural to expand it in the Fourier series, but if we regard the circle as an interval, we may use the Fourier transform as well.

We derive the scaling model by studying the behavior of a rectangular (indicator) function at the north pole, and subsequently show how it can be used to model arbitrary axisymmetric and separable nonaxisymmetric functions. By axisymmetric functions we refer (somewhat imprecisely) to functions that depend only on the

Copyright (c) 2010 IEEE. Personal use of this material is permitted. However, permission to use this material for any other purposes must be obtained from the IEEE by sending a request to pubs-permissions@ieee.org.

This work was supported by Ministry of Science, Education and Sports of Croatia under Grants MZOS 0036054 and MZOS 036-0362214-1987.

Authors are with the Department of Electronic Systems and Information Processing, Faculty of Electrical Engineering and Computing, University of Zagreb, Unska 3, 10000 Zagreb e-mails: ivan.dokmanic@fer.hr, davor.petrinovic@fer.hr. colatitude $\theta$. This restriction simplifies the derivation of the scaling model. We demonstrate the applicability of the derived model to separable nonaxisymmetric functions in Section V. The derived result is strengthened by the fact that it has a straightforward higherdimensional generalization (i.e. generalization to functions that live on an $n$-sphere).

\section{MODEL}

The classical Fourier transform of a scaled function on the real line $f(a x)$ is $|a|^{-1} F(\omega / a)$ where $F(\omega)=\int f(x) e^{-j \omega x} d x$. We propose a comparably simple model for scaling of functions on the 2-sphere $S^{2}$. Let $f \in L^{2}\left(S^{2}\right)$. Its spectrum is given by $F(l, m)=$ $\int_{S^{2}} f Y_{l}^{m} d \omega$ where

$$
Y_{l}^{m}(\theta, \phi)=\sqrt{\frac{2 l+1}{4 \pi} \frac{(l-m) !}{(l+m) !}} P_{l}^{m}(\cos \theta) e^{j m \phi}
$$

are the spherical harmonics of order $l$ and degree $m$ and $d \omega=$ $\sin \theta d \theta d \phi$ is the rotation invariant measure in the usual spherical polar coordinates [9], [10]. $P_{l}^{m}$ are the associated Legendre functions [9] which degenerate to Legendre polynomials $P_{l}$ for $m=0$. Since we deal with an axisymmetric $f, F(l, m)=0$ for $m \neq 0$ i.e. its spatial and spectral representations are fully determined by one dimensional signals and sequences.

Furthermore, let $g(\theta, \phi)=f(k \theta, \phi), k>0$. Then for some exponent $\beta$ we would like to compute the spectrum of $g$ as

$$
G(l) \equiv G(l, 0)=F(l / k, 0) \cdot k^{-\beta} .
$$

We omit the second spectrum index $m$ since in the derivation of the model we deal exclusively with axisymmetric functions. Note that (2) generally requires evaluation of $F$ at the non-integer argument. It anticipates the (hypothetical) underlying continuous spectrum that will be used to derive the scaling model.

This correspondence demonstrates that such a simple model is only approximate, but yields a good solution for a certain class of functions. Furthermore, in deriving the model we show that the notion of continuous spectrum naturally follows from hypergeometric generalization of Legendre and Gegenbauer polynomials [9].

\section{A. Rect function}

The goal of this subsection is to determine the exponent $\beta$ in (2). The technique that follows is inspired by two assumptions:

1) The model, though approximate, should be the same for all spherical signals.

2) The model should be applicable for all scaling factors, even very small i.e. the model must also be valid in the $k \rightarrow 0$ limit.

Given these, we find the $\beta$ for the simplest case - a rectangular function on a 2 -sphere - and then apply it to other signals. 
Denote by $r_{a}$ a spherical analogue of a rect function centered around the north pole. It is defined as

$$
r_{a}(\theta, \phi)= \begin{cases}1 & \text { for } \theta \leq a \\ 0 & \text { otherwise }\end{cases}
$$

with spectrum given by

$$
R_{a}(l)=\frac{\sqrt{\pi}\left(P_{(l-1)}(\cos a)+P_{(l+1)}(\cos a)\right)}{\sqrt{2 l+1}} .
$$

Note that we call it a "rect" function since it is a rectangle when evaluated along the meridian.

According to the proposed scaling model (2), we would like the following to hold for some $\beta$,

$$
R_{a}(l)=R_{(k a)}(l / k) \cdot k^{-\beta},
$$

which follows from (2) and the fact that $r_{a}(\theta, \phi)=r_{(k a)}(k \theta, \phi)$. It is assumed here that we can somehow evaluate (4) for non-integer $l$. It is indeed possible to do it if we express the Legendre polynomial in terms of a hypergeometric function ${ }_{2} F_{1}$, see for example [9]. The behavior of (5) may be better understood if we expand the right hand side in Taylor series about $a=0$,

$$
R_{a k}(l / k) \cdot k^{-\beta}=\frac{k^{1.5-\beta}}{\sqrt{2 l+k}} \sum_{r=1}^{\infty} \operatorname{poly}_{2 r-1}(l, k) a^{2 r}
$$

where $\operatorname{poly}_{2 r-1}(l, k)$ is a complete homogeneous polynomial of order $2 r-1$.

Ideally, a single solution for $\beta$ (a single model) would be suitable for any choice of $a, l$ and $k$. Since the model is approximate, the exact solution for $\beta$ varies with these parameters. We would like to somehow choose a generally suitable $\beta$. Since the model equality (5) must be valid for all $k>0$, we require it to be correct in the $k \rightarrow 0$ limit as well. It is clear from (6) that we must choose $\beta=\frac{3}{2}$ in order for $\lim _{k \rightarrow 0} R_{a k}(l / k) \cdot k^{-\beta}$ not to vanish or diverge. Any other choice would result in the wrong amplitude of a spatially scaled function when contracted using the proposed model.

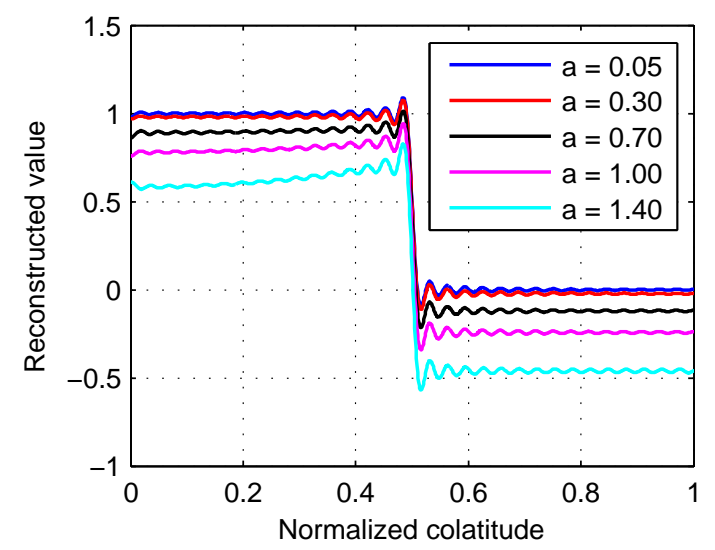

Fig. 1. Rectangle-like functions obtained by inverse transform of the "limit" spectrum (7) plotted along the meridian. Bandwidth used for the narrowest $a=0.05$ case was 2000 . Bandwidths for other cases are accordingly scaled (inversely proportional). Abscissae are normalized so that the discontinuity occurs at 0.5. This two facts result in a matching ripple frequency for all widths.
It is informative to compute this limit explicitly,

$$
\begin{aligned}
\lim _{k \rightarrow 0} R_{a k}(l / k) \cdot k^{-\beta} & =a^{2} \sqrt{\frac{\pi l}{2}} \sum_{r=0}^{\infty} \frac{(-1)^{r}}{r !(r+1) !}\left(\frac{a l}{2}\right)^{2 r} \\
& =a \sqrt{2 \pi / l} J_{1}(a l),
\end{aligned}
$$

where $J_{1}$ is the Bessel function of the first kind and order one (see e.g. [9]).

Fig. 1 shows the inverse transform of the sampled continuous spectrum (7) plotted along the meridian, with scaled abscissae. Ideally, this would give identical bandlimited rectangular signals, but due to approximation, we get the result that depends on $a$. Note however that it perfectly models the discontinuity at $a(0.5$ with scaled abscissa). Furthermore, note that this is simply a plot of the inverse transform of (7), not the actual application of the model.

Actual (analytical) rectangle spectrum (4) in comparison to the limit spectrum (7) is shown in Fig. 2. Even though a slight mismatch may be noticed for spatially wider functions, the similarity is significant.

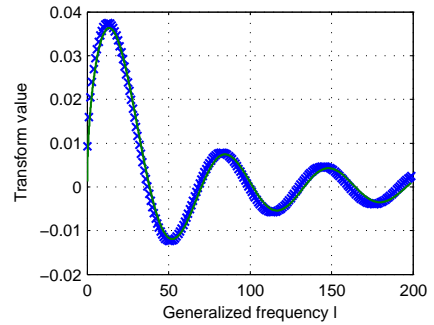

(a)

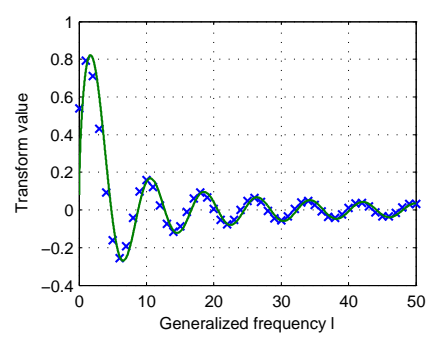

(c)

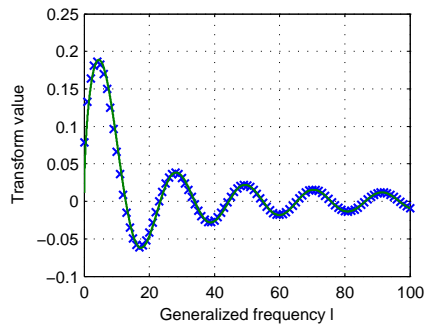

(b)

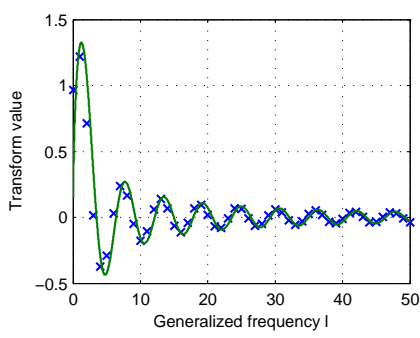

(d)
Fig. 2. Discrete spectrum of the spherical rect signal shown against the limit continuous spectrum given by (7) for different widths $a$. (a) $a=0.1$, (b) $a=0.3$, (c) $a=0.8$, (d) $a=1.1$.

\section{III. $n$-SPHERE}

The described approach may be generalized to $n$-dimensional spheres embedded in $\mathbb{R}^{n+1}$. Using the usual hyperspherical angular coordinates (see for example [5], [10]) we may write the hyperspherical harmonics on $S^{n}$ as [10], [11],

$\Xi_{\mathbf{K}}^{l}(\xi)=A_{\mathbf{K}}^{l} \prod_{i=0}^{n-2} C_{k_{i}-k_{i+1}}^{\frac{n-i-1}{2}+k_{i+1}}\left(\cos \theta_{n-i}\right) \sin ^{k_{i+1}} \theta_{n-i} e^{ \pm j k_{n-1} \theta_{1}}$

A compound index $\mathbf{K}$ is a multi-index $\left(k_{1}, \ldots, k_{n-1}\right)$ of integers such that $l \equiv k_{0} \geq k_{1} \geq \cdots \geq k_{n-2} \geq\left|k_{n-1}\right| \geq 0 . C_{p}^{\lambda}(t)$ are the Gegenbauer polynomials [9] and the normalization factor is given by 


$$
\begin{aligned}
\left(A_{\mathbf{K}}^{l}\right)^{2} & =\frac{1}{2 \pi^{(n+1) / 2}} \prod_{i=0}^{n-2} 2^{2 k_{i+1}+n-i-3} \times \\
& \times \frac{\left(k_{i}-k_{i+1}\right) !\left(n-i+2 k_{i}-1\right) \Gamma^{2}\left(\frac{n-i-1}{2}+k_{i+1}\right)}{\sqrt{\pi} \Gamma\left(k_{i}+k_{i+1}+n-i-1\right)} .
\end{aligned}
$$

The generalized axial symmetry means that the signal is dependent on one angle parameter only and that the spectrum may be indexed by a single index $l$, same as for the $n=2$ case. For an axisymmetric function $f$ we compute the Fourier coefficients as

$$
F(l)=\int_{S^{n}} f(\omega) \Xi_{\mathbf{0}}^{l}(\omega) d \omega
$$

where subscript $\mathbf{0}$ denotes the multi-index $\mathbf{K}=(0, \ldots, 0)$ and

$$
d \omega=\prod_{j=1}^{n} \sin ^{n-j} \theta_{n-j+1} d \theta_{j}
$$

is the rotation invariant measure on $S^{n}$ in hyperspherical coordinates.

We may compute the rectangle spectrum and the limit spectrum analogously to the 2 -sphere case. The derivation is algebraically more involved than in the 2-sphere case but the presented final results are again simple. Derived results for sphere dimensionalities $n=2,3$ and 4 are summarized in Table I. One can assume that $\beta$ as introduced in (2) is always $(n+1) / 2$ on an $n$-sphere, but we don't prove this.

\section{TABLE I}

SERIES TERMS AND LIMITS OF THE RECTANGLE SPECTRUM SCALED WITH THE SCALE FACTOR $k \rightarrow 0$ FOR DIFFERENT DIMENSIONALITIES.

\begin{tabular}{lccc}
\hline$n$ & $\begin{array}{c}r \text {-th series term } \\
\left(\sum_{r=0}^{\infty}\right)\end{array}$ & $\begin{array}{c}\text { Series sum } \\
\text { (cont. spectrum) }\end{array}$ & $\begin{array}{c}\text { Scaling exp. } \\
\beta\end{array}$ \\
\hline 2 & $\sqrt{\frac{\pi l}{2} \frac{(-1)^{r}(l / 2)^{2 r}}{r !(r+1) !} a^{2+2 r}}$ & $a \sqrt{\frac{2 \pi}{l}} J_{1}(a l)$ & $\frac{3}{2}$ \\
3 & $2 l \sqrt{2} \frac{(-1)^{r}\left(l^{2} / 2\right)^{r}}{r !(3+2 r) ! !} a^{3+2 r}$ & $2 a^{\frac{3}{2}} \sqrt{\frac{\pi}{l}} J_{\frac{3}{2}}(a l)$ & $\frac{4}{2}$ \\
4 & $\left(\frac{l}{2}\right)^{\frac{3}{2}} \frac{\pi(-1)^{r}(l / 2)^{2 r}}{r !(r+2) !} a^{4+2 r}$ & $\pi a^{2} \sqrt{\frac{2}{l}} J_{2}(a l)$ & $\frac{5}{2}$
\end{tabular}

The limit spectrum may be further rewritten using the spherical Bessel function $j_{p}(x)=\sqrt{\pi / 2 x} J_{p+1 / 2}(x)$. This fact is very interesting since the spherical Bessel function is related to the $\sin x / x$ (sinc) function, and it is known that the spectrum of a rectangle in the Euclidean case is in fact a sinc [12]. In particular,

$$
j_{p}(x)=(-x)^{p}\left(\frac{1}{x} \frac{d}{d x}\right)^{p} \frac{\sin x}{x} .
$$

For integer $p$ this results in a combination of damped sines and cosines. This is actually the case for odd-dimensional spheres embedded in even-dimensional Euclidean spaces e.g. $S^{3} \subset \mathbb{R}^{4}$. Consequently, the series sum for $n=3$ in the second row of Table I may also be written as $2 l \sqrt{2}(\sin a l-a l \cos a l) / l^{2}$.

\section{EXPERIMENT}

Since the proposed model is one-parametric, it is completely determined by the derived choice of $\beta$. We now apply it to different signals. The setup is as follows - we first compute the mother spectrum and then resample it to find the approximate spectrum of a scaled signal (thus assuming the existence of the underlying continuous spectrum). Note that we use simple linear interpolation for resampling. If the scaling asks for bandwidth expansion (resampling on a denser grid) then linear may be replaced by some more involved interpolation scheme, such as cubic spline. Nevertheless, linear interpolation should suffice for relatively smooth spectra.

The scaling algorithm is summarized in Algorithm 1. It is exactly what was used to generate the plots in Fig. 3. Note that this algorithm automatically adapts the spectrum width to the scaled signal width in the spatial domain.

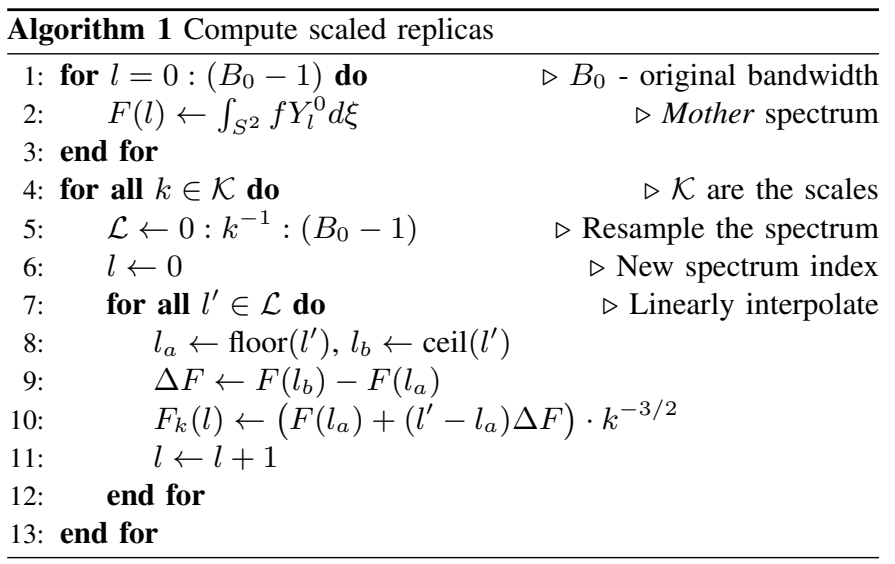

One can verify that the simple linear interpolation works well in Fig. 3. This figure was obtained by scaling the functions with the proposed scaling model and than exactly rescaling them back to enable a meaningful comparison. It is also observable that the model works better for functions whose energy is concentrated near the north pole. This is intuitive given that the Taylor expansion (6) is about $a=0$, where $a$ is the width of the rect function. Fig. 4 shows the spherical plots of the rectangle and Gaussian-like function scaled using the proposed model for different scaling factors.

Furthermore, note that we do not include the inverse transform in the Algorithm 1. This is because the whole idea is to operate in the spectral domain directly. Indeed, many applications do not require the inverse transform up until late in the process (see for example [5]).

\section{A. Approximation error}

We have used two signals for model validation: a bandlimited version of a spherical rectangle defined in (3), and a smooth, Gaussian-like function given by

$$
f_{s}(\theta)=e^{-(\theta / s)^{2}}, \quad \theta \in[0, \pi] .
$$

The approximation errors were computed as

$$
E(k)=10 \log _{10} \frac{\int_{0}^{\theta_{\max }}\left\{f(\theta)-\widetilde{f}_{k}(\theta)\right\}^{2} d \theta}{\int_{0}^{\theta_{\max }} f(\theta)^{2} d \theta} .
$$

The function $\tilde{f}_{k}$ was obtained by scaling $f$ with a scale factor $k$ using the proposed model and then scaling it back with a scale factor $1 / k$ using the exact spatial scaling. In our experiments $\theta_{\max }$ corresponds to unit value of the normalized colatitude on the plots in Fig. 3.

The error curves shown in Fig. 3 suggest that the smoother functions like the Gaussian exhibit smaller approximation errors over a wider range of scaling factors. The dashed line in Fig. 3f shows the error for cubic spline based resampling instead of linear. It can be observed that the error becomes saturated as we contract the function, but becomes large when the function is dilated (approximately when the scaled function occupies more than a half of the sphere). 


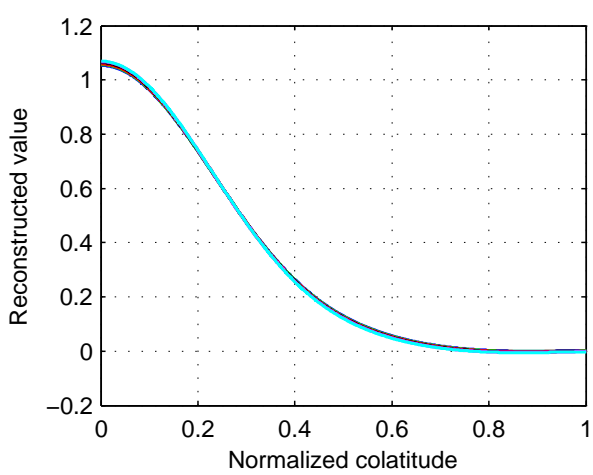

(a)

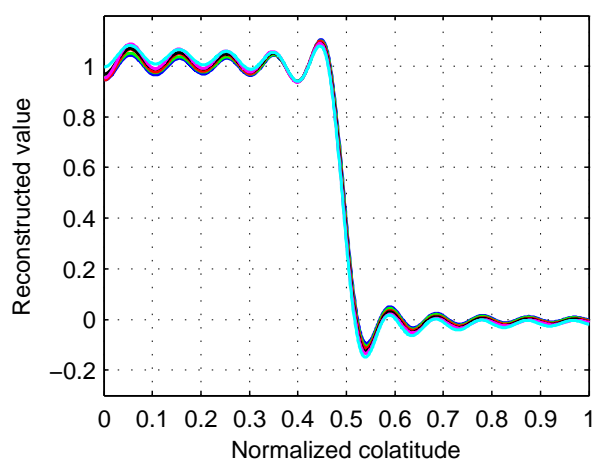

(d)

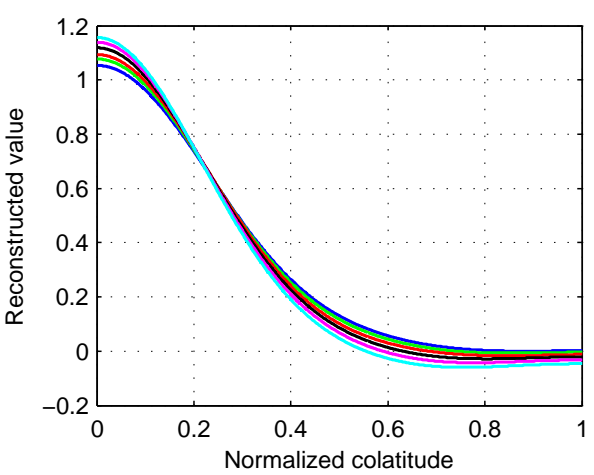

(b)

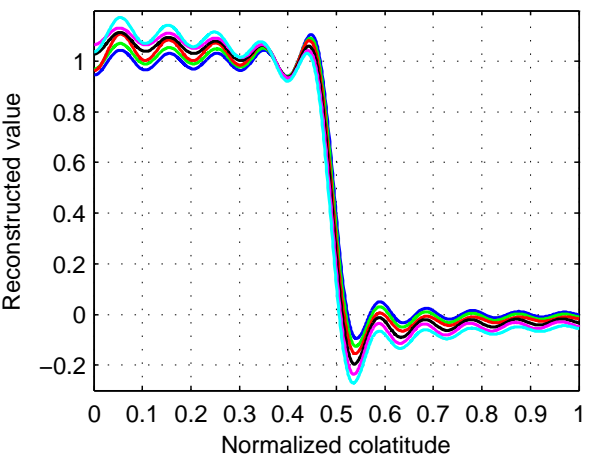

(e)

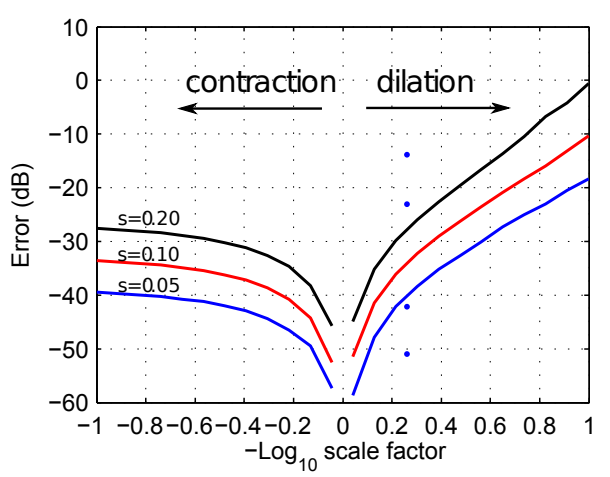

(c)

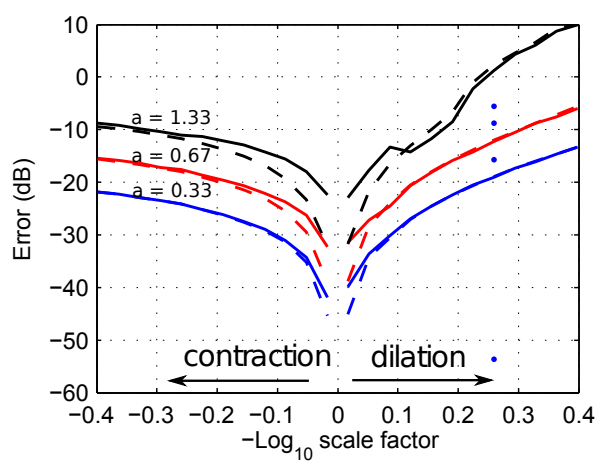

(f)

Fig. 3. Functions on a sphere approximately scaled using the proposed spectral model. Subfigures (a) and (b) show the scaling of the Gaussian-like function (12) for scaling ranges $k \in[1,2.5]$ and $k \in[1,11]$. with initial $s=0.05$. (d) and (e) show the scaling of a bandlimited rect signal (3) for scaling ranges $k \in[1,1.5]$ and $k \in[1,2.5]$ with initial $a=0.33$. (c) and (f) show the error (13) due to approximation for both contracted and dilated cases. Dashed line in (f) shows the error with cubic spline interpolation instead of linear. For Gaussian, $k \in[0.1,10]$ and for rect, $k \in[0.4,2.5]$. In (c) and (f), round dots show the errors obtained with the method proposed in [4] for $s=0.05$ and $a=0.33$, respectively. The case with the smallest error was computed using the exact matrix for $k=0.55$. Other cases were computed by interpolating between matrices for $(k=0.5$ and $k=0.6),(k=0.4$ and $k=0.7)$ and $(k=0.3$ and $k=0.8)$.

The approximation error depends on the function being scaled, its initial scale and on the scaling factor itself. A more detailed theoretical error analysis would be very complicated and falls outside of the scope of this manuscript. Numerically computed errors in Fig. 3 suggest that the proposed method is applicable in applications that don't require numerical exactness, but involve subjective (i.e. visual) assessment of the scaled function, as can be seen in Fig. 4. Note that in the bandlimited scenario, scaling always involves an approximation, regardless of the used scaling method.

\section{EXTENSION TO SEPARABLE FUNCTIONS}

Even though we claimed that we will discuss functions that depend only on $\theta$, we will outline the extension of the model to separable nonaxisymmetric functions. These are the functions that can be written as a product of azimuthal and colatitudal part, $f(\theta, \phi)=u(\theta) v(\phi)$. The key insight here is given by Fig. 5 and the observation that

$$
\begin{aligned}
F(l, m) & =N_{l}^{m} \int u(\theta) P_{l}^{m}(\theta) \sin \theta d \theta \int v(\phi) e^{j m \phi} d \phi \\
& =A(l, m) e^{j \Phi(m)},
\end{aligned}
$$

where $N_{l}^{m}$ is the normalization factor as in (1). In (14), $\Phi(m)$ represents the total phase of the second integral, which is also the total phase of $F(l, m)$ since the first integral has a zero phase. For this reason, the phase of $F(l, m)$ depends only on $m$ (the integral with respect to $\phi$ doesn't depend on $l$ ). This in turn corresponds

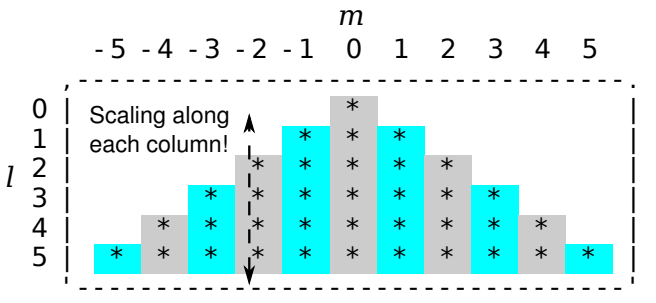

Fig. 5. Shape of the spectrum of a bandlimited spherical function. The arrow indicates the direction of the scaling in spectrum.

to the fact that each column in Fig. 5 has a constant phase in the separable case. The algorithm then proceeds as follows (for each column of the spectrum): (1) decouple amplitude and phase, (2) interpolate the amplitude according to Algorithm 1, (3) reintroduce the phase information. Fig. 6 shows the result of the application of this procedure to a separable spherical function.

\section{COMPARISON WITH AN EXISTING METHOD}

It was noted by authors in [4] that the scaling of spherical functions may be regarded as a linear operator that acts on the vector of spherical harmonic coefficients. This method essentially provides exact scaling, with the remarks that follow. Elements of the operator matrix are computed using numerical integration. The cost of this computation prohibits the real-time computation of matrix elements. They must be computed in advance for some scaling factors and 


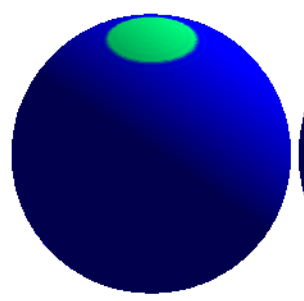

(a)

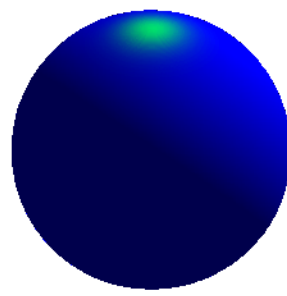

(e)

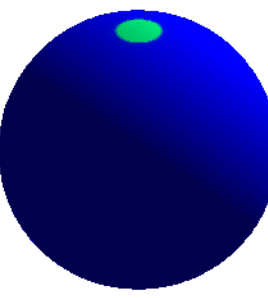

(b)

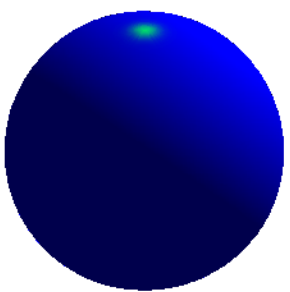

(f)

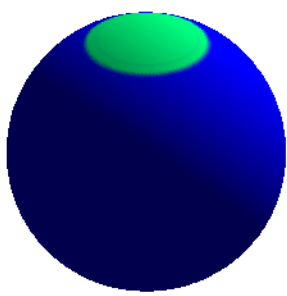

(c)

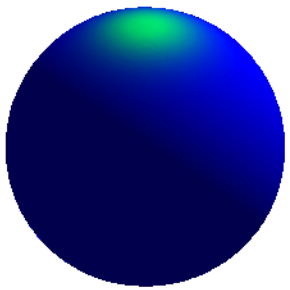

(g)

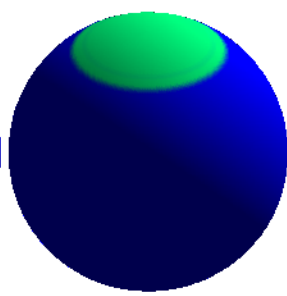

(d)

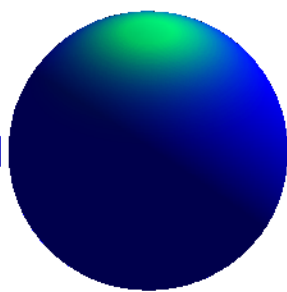

(h)

Fig. 4. Spherical plots of the functions scaled using the proposed model: (a) mother bandlimited rectangle (3) with $a=0.33$, (b)-(d) rectangle in (a) scaled with scaling factors $k^{-1}=0.5,1.4,1.8$, (e) mother bandlimited Gaussian-like function (12) with $s=0.25$, (f)-(h) Gaussian in (e) scaled with scaling factors $k^{-1}=0.4,1.6,2.4$.

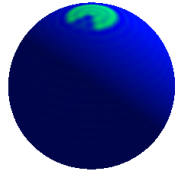

(a)

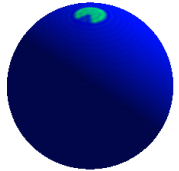

(b)

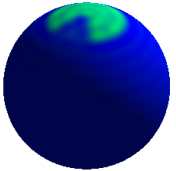

(c)

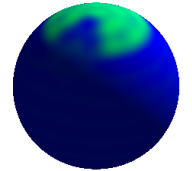

(d)
Fig. 6. Spherical plots of the separable "mother" function in (a) scaled by factors (b) $k^{-1}=0.5$, (c) $k^{-1}=1.7$, (d) $k^{-1}=2.4$ using the proposed separable extension.

then interpolated for other, arbitrary factors. Furthermore, scaling of the bandlimited function generally produces a non-bandlimited function, therefore their method is approximate as well. However, it may be made arbitrarily exact whereas the model proposed in this paper always yields an approximation.

If we are scaling a function $f(\theta, \phi)$ with bandwidth $B$ by a factor $k, f_{k}(\theta, \phi)=f(k \theta, \phi)$, the model that we propose assumes a proportional bandwidth scaling as well. That is, if the function $f_{k}$ is approximated using the proposed model, its bandwidth is $k \cdot B$. Since the complete spherical Fourier transform of a spherical function with the bandwidth $B$ has $B^{2}$ samples, and given that the complexity of linear interpolation is proportional to the number of output samples, it is easy to see that the proposed method has a complexity of $O\left(k^{2} B^{2}\right)$.

On the other hand, the method proposed in [4] involves multiplying the coefficient vector of size $B^{2}$ by a scaling matrix of size $B^{2} \times$ $B^{2}$. This matrix, however, is a sparse matrix, with only $B\left(2 B^{2}+\right.$ $1) / 3$ non-zero entries. Therefore, the complexity of their method is $O\left(k^{3} B^{3}\right)$ (again by assuming the appropriate bandwidth scaling).

The method proposed in [4] requires the scaling matrices to be precomputed. If the application calls for a relatively high bandwidth, storing these matrices may become a problem. As an example, if we wanted to store 40 scaling matrices of bandwidth $B=200$ using a double precision, we should allocate around $1.7 \mathrm{~GB}$ of memory for non-zero matrix elements. This problem becomes more prominent if we want to shrink the function, since this generally requires bandwidth expansion. Scaling the function with $k=3$ would require $46 \mathrm{~GB}$ of memory for precomputed matrices in the above example. In contrast, the property that we exploit in this paper has no storage requirements except for the input and output transform coefficients. At $B=200$ and double precision, spherical coefficients occupy $320 \mathrm{~KB}$ of memory.

To compare the accuracy of the two methods, we have computed the scaling matrices for the scaling factor $k=0.55$ and for several nearby scale factors. Errors were computed using (13), and are denoted in the Fig. 3c and $3 \mathrm{f}$ by round dots (corresponding to the lowermost error curves). For the smallest error, the exact matrix was used, and for the other three cases, the scaling matrix was obtained by linear interpolation. In the exact case the baseline error is determined by the accuracy of matrix elements (numerical integration on an equiangular grid with 160000 knots). Furthermore, the scaled function is never exactly bandlimited and the spectrum truncation also introduces an error. We may conclude that if the scaling matrix is computed exactly, then the accuracy of the method in [4] is significantly higher than the accuracy of the method proposed in this paper. However, in the real-time applications only the finite number of scaling matrices may be precomputed in which case the errors may become comparable.

The final issue is generality. Property described in this paper is readily applicable only for axisymmetric functions and separable nonaxisymmetric functions. Generalization is the topic of an ongoing research. On the other hand, it is easily extended to $n$-spheres which is of interest for higher dimensional applications whereas only the 2-sphere case is considered in [4].

\section{CONCLUSION}

We have derived and demonstrated a simple spectral domain based technique for scaling of the functions on an $n$-sphere. Although this technique provides approximate scaling, the results suggest that it may be used in many practical situations where efficiency and simplicity are required.

In summary we have shown that the Fourier transform of axisymmetric function $f(k \theta)$ on an $n$-sphere is approximately $k^{-(n+1) / 2} F(l / k)$, where $F(l)$ is the Fourier transform of $f(\theta)$ and $F$ is evaluated at the non-integer positions in a resampling sense. This result is analogous to the Euclidean case, and in fact degenerates to the familiar $|a|^{-1} F(\omega / a)$ if we set $n=1$ in the previous expression. 
We have also outlined the extension of the algorithm to separable spherical functions that are not axisymmetric and provided a comparison with an existing scaling method.

Further work involves extension to nonseparable, nonaxisymmetric functions.

\section{REFERENCES}

[1] J. p. Antoine and C. B. Torresani, "Wavelets on the 2-sphere: A grouptheoretical approach," Appl. Comput. Harmon. Anal, vol. 7, pp. 1-30, 1999.

[2] J. Antoine and P. Vandergheynst, "Wavelets on the 2-sphere and related manifolds," Reports on Mathematical Physics, vol. 43, no. 1-2, pp. 1324, 1999.

[3] B. Yeo, W. Ou, and P. Golland, "On the construction of invertible filter banks on the 2-sphere," IEEE Trans. Image Process., vol. 17, no. 3, pp. 283-300, March 2008.

[4] J. Wang, K. Xu, K. Zhou, S. Lin, S. Hu, and B. Guo, "Spherical harmonics scaling," The Visual Computer, vol. 22, no. 9, pp. 713-720, Sep. 2006. [Online]. Available: http://dx.doi.org/10.1007/s00371-0060057-8

[5] I. Dokmanic and D. Petrinovic, "Convolution on the $\mathrm{n}$-sphere with application to pdf modeling," Signal Processing, IEEE Transactions on, vol. 58, no. 3, pp. $1157-1170$, March 2010.

[6] P. Hall, G. S. Watson, and J. Cabrera, "Kernel density-estimation with spherical data," Biometrika, vol. 74, no. 4, pp. 751-762, Dec. 1987.

[7] J. R. Driscoll and J. Dennis M. Healy, "Computing Fourier transforms and convolutions on the 2-sphere," Adv. Appl. Math., vol. 15, no. 2, pp. 202-250, 1994.

[8] D. Healy, D. Rockmore, P. Kostelec, and S. Moore, "FFTs for the 2-sphere-improvements and variations," Journal of Fourier Analysis and Applications, vol. 9, no. 4, pp. 341-385, Jul. 2003. [Online]. Available: http://dx.doi.org/10.1007/s00041-003-0018-9

[9] I. Gradshteyn and I. Ryzhik, Table of Integrals, Series, and Products, 7th ed., A. Jeffrey and D. Zwillinger, Eds. Academic Press, Inc., 2007.

[10] N. Y. Vilenkin, Special functions and the theory of group representations, 1st ed. American Mathematical Society, 1968.

[11] J. Avery, Hyperspherical Harmonics: Applications in Quantum Theory, 1st ed., ser. Reidel Texts in the Mathematical Sciences. Springer, 1989, vol. 5.

[12] A. V. Oppenheim, R. W. Schafer, and J. R. Buck, "Discrete-time signal processing (2nd ed.)," 1999. 